\author{
Proceedings of the $10^{\text {th }}$ International Conference on Applied Informatics \\ Eger, Hungary, January 30-February 1, 2017. pp. 95-102 \\ doi: 10.14794/ICAI.10.2017.95
}

\title{
Cloud Service Architecture Optimization Methodology for Telemedicine and e-Health Interoperability Framework
}

\author{
Ábel Garai \\ University of Debrecen \\ garai.abel@inf .unideb.hu
}

\begin{abstract}
Mobile healthcare appliances' bulk interconnection is substantially beneficial both on individual and on population level resulting in enhanced healthcare prognosis models. The flawless mobile healthcare information-exchange on larger scale calls for improved healthcare data interchange standards and suitable architectural design. The challenges of healthcare interoperability standards were thoroughly discussed in previous articles. As a next step, this article focuses on the mathematical methodology for e-Health service architecture optimization. In general, this research program targets to establish the adequate scientific framework for universal cloud-based e-Health biosensory devices interconnection. In particular, this paper focuses specifically on the underlying cloud service architecture's optimization. Statistical optimization methodologies already existed for system structure improvement. However, this presented optimization procedure delivers the required comprehensive solution applicable also on complex hybrid systems. This methodology sets adequate procedures for comparing complex system landscapes. This procedure derives directed graphs from the system design-layout and maps them into adjacency matrices for matrix algebra analysis. Finally, the comparison of the derived availability matrices results in the identification of the optimal architecture design. This technique offers the appropriate mathematical methodology for the cloud-based e-Health service architecture optimization. This demonstrated methodology delivers the appropriate system framework. This is required for the optimal interconnection of the e-Health smart device, telemedicine instrument and hospital information system subdomains.
\end{abstract}

Keywords: Telemedicine, cloud architecture optimization, matrix algebra, adjacency matrix, availability matrix, directed graph

$M S C$ : Primary 68R10; Secondary 68-02. 


\section{Introduction}

The actual information technology megatrends predestinate the collective real-time exchange and exploration of healthcare data. The four most influential international tendencies are the spread of cloud technologies [1] and the Internet-of-Things [2] concept, the exponential market-penetration of eHealth [3] smart devices [4]; and the arising Big Data [5] analysis capabilities. However, the healthcare information systems, telemedicine devices and e-Health appliances operate still separately from each other. This research's vision is to specify, plan, develop, test, deploy and integrate the appropriate centralized, cloud-based open telemedicine interoperability architecture-framework. For the aforementioned purpose, this paper specifies the methodology for the comparison and selection of the best suitable cloud-based telemedicine architectural design. The general research goal is the universal interoperability of different e-Health smart devices, telemedicine instruments and healthcare information systems. Therefore, the ideal healthcare interoperability service architecture needs to be systematically specified. According to the presented methodology, the optimized service architecture is selected through comparison of different system landscapes. This paper focuses specifically on this dedicated mathematical methodology for the selection of the optimal service architecture landscape.

\section{Research background}

This research is the manifestation of the cooperation among the University of Debrecen Faculty of Informatics, Semmelweis University 2nd Peadiatric Clinic Department of Pulmonology and two healthcare industry-partners. The exploration team focused on establishing universal interoperability interconnecting the different healthcare devices and systems with each other. The physical examination was conducted on e-Health smart wearable devices, a clinical spirometer and a hospital information factory test system. The open telemedicine interoperability hub-software (OTI-HS) was specified [6], developed, tested, deployed [7] and integrated [8] in cloud-architecture. The specified system solution intercepts, interprets, converts, stores, aggregates and dispatches healthcare bio-sensory dataflow among diverse healthcare devices, technologies and hospital information systems. The final, optimized system architecture is embedded in hybrid cloud.

\section{Related Works}

Gill et al published numerical linear algebra optimization methodology as early as in the 1980s [9]. Their methodology is generally applicable in multiple scientific areas. Otto et al defined a wireless body area sensor network system architecture for health monitoring [10]. Carson stipulates the key elements in this research field: quantifying system complexity for global system architecture optimization 
[11]. The topic is followed by Fischi et al more specifically with regard to dynamic complexity measurement for system design [12]. Pokorádi developed graph-based system modelling framework and applied it for complex technical system-analysis $[13]$.

\section{Purpose and target-setting}

The research program's initial hypothesis is that the optimal system architecture layout is designed through a centralized, cloud-based, open telemedicine interoperability hub-software. The presented methodology delivers the tool-set for the justification of the initial hypothesis' correctness. It selects the optimal cloudbased telemedicine architecture design-concept through matrix-algebra operations. This method forms abstract, mathematical representation of the system architecture design-concepts, performs defined matrix algebra operations on them, conducts comparison of the results, and selects the optimal system layout based on the mathematical results. The method is structured into the following six-steps:

1. Map each service-oriented e-Health cloud-architecture design-concept into associated directed graph,

2. Map the directed graphs into adjacency matrices,

3. Calculate the availability matrices derived from the adjacency matrices,

4. Calculate all elements' sum for each availability matrix,

5. Sort the resulting avialability matrices based on their all elements' sums ascending, and

6. Select the optimal telemedicine architecture layout according to the highest availability matrix all element sum.

\section{Discussion}

This research program defines a universal cloud-architecture for telemedicine and e-Health interoperability. This architecture has a central element: the OTI-HS (Figure 1./5/). This component interconnects the different technology domains with each other: the e-Health smart device consumer electronics and their smartphone app-client (Figure 1./1 \& 2/), the clinical telemedicine instruments and their client-software (Figure 1./3\& $4 /$ ), the hospital information systems and their wireless user-interface (Figure $1 . / 6 \& 7 /$ ).

All these components are interconnected through the central OTI-HS (Figure 1.). However, there are only unidirectional network connections between only some architecture components at the beginning of the research. The research hypothesis is that the ideal cloud-based telemedicine architecture should rely upon a central 
system component (represented by the OTI-HS, Figure 1. /5/). This methodology justifies, that the OTI-HS-based architecture design is the optimal one, because that maximizes the number of direct links among the system components. The designed system landscape is mapped into directed graph for the hypothesis' mathematical verification (Figure 1.)

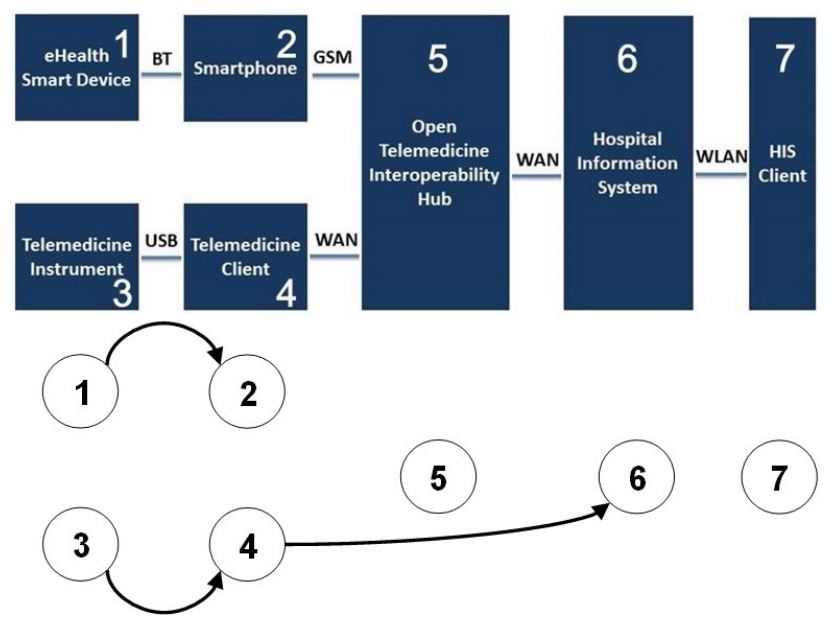

Figure 1: Cloud-based open telemedicine inteoperability architecture-landscape and its mapped directed graph

Three different cases are represented and validated in this paper: the initial research phase, when the individual system components are functioning in "silo" mode, separated from each other. At the beginning of the research the eHeath device had only unidirectional communication connection with its factory smartphone-app, and also the telemedicine instrument communicated only with its client software. The research's aim is, that all of these system components should have communication link with each other. Therefore, there are only three edges in Case A's graph (Figure 2.). During the research program, the OTI-HS established additional connections with the e-Health device, with the telemedicine instrument and with the hospital information system, as shown in B (Figure 2. Case B.). Therefore, there are significantly more edges on Case B's graph than on Case A's graph. The 3rd case (Figure 2. Case C.) illustrates the optimal scenario, when beyond unidirectional connection, bidirectional connections are established among most of the system devices and instruments. Therefore, the Case C's graph has more edges than Case B's and Case A's.

The mathematical methodology consists of the steps as follows. 


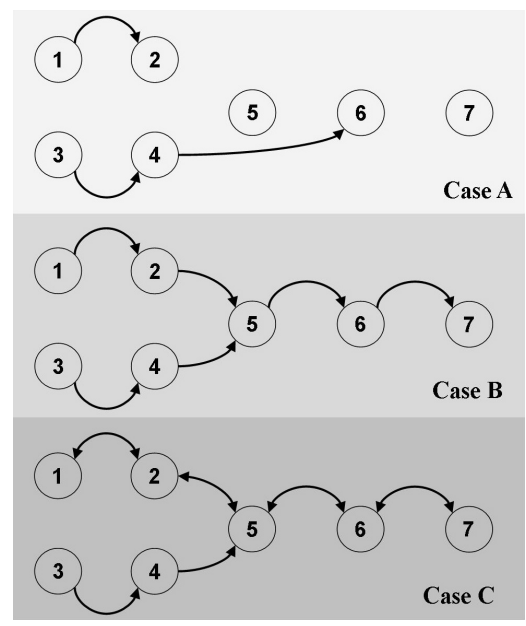

Figure 2: System layout scenarios. Case A: Legacy / Case B:

Experimental / Case C: Optimal

Step 1. We map Case C's directed graph into its corresponding adjacency matrix $(\mathrm{C})$ :

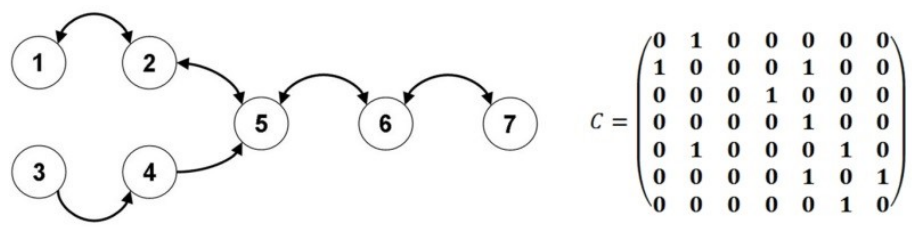

Step 2. In order to calculate the availability matrix for Case C, we sum up the powers of its adjacency matrix (C) based on Formula I:

$$
H_{k}=\sum_{(n-1)}^{k} A^{n}
$$

Formula I. returns $H_{C}$ interim-matrix:

$$
H_{C}=H_{7}=\left[\begin{array}{ccccccc}
8 & 22 & 0 & 0 & 13 & 18 & 5 \\
22 & 21 & 0 & 0 & 40 & 18 & 18 \\
4 & 13 & 0 & 1 & 9 & 13 & 4 \\
13 & 13 & 0 & 0 & 27 & 13 & 13 \\
13 & 40 & 0 & 0 & 26 & 40 & 13 \\
18 & 18 & 0 & 0 & 40 & 21 & 22 \\
5 & 18 & 0 & 0 & 13 & 22 & 8
\end{array}\right]
$$


Step 3. We apply the signum function to the $H_{C}$ interim-matrix:

$$
S_{k}=\operatorname{sign} H_{k}=\operatorname{sign} \sum_{(n-1)}^{k} C^{n}
$$

Thereby we obtain the availability matrix $\left(S_{C}\right)$ for Case C:

$$
S_{C}=S_{7}=\left[\begin{array}{lllllll}
1 & 1 & 0 & 0 & 1 & 1 & 1 \\
1 & 1 & 0 & 0 & 1 & 1 & 1 \\
1 & 1 & 0 & 1 & 1 & 1 & 1 \\
1 & 1 & 0 & 0 & 1 & 1 & 1 \\
1 & 1 & 0 & 0 & 1 & 1 & 1 \\
1 & 1 & 0 & 0 & 1 & 1 & 1 \\
1 & 1 & 0 & 0 & 1 & 1 & 1
\end{array}\right]
$$

We obtain all elements' sum for Case C's (AM's) availability matrix as we multiply it with the transposed sum vector from the left, and with the sum vector from the right:

$$
X_{C}=\underline{1}^{T} \cdot S_{7} \cdot \underline{1}
$$

Therefore, all elements' sum (SM) for Case C's (AM's) availability matrix is:

$$
X_{C}=\left[\begin{array}{lllllll}
1 & 1 & 1 & 1 & 1 & 1 & 1
\end{array}\right] \cdot\left[\begin{array}{lllllll}
1 & 1 & 0 & 0 & 1 & 1 & 1 \\
1 & 1 & 0 & 0 & 1 & 1 & 1 \\
1 & 1 & 0 & 1 & 1 & 1 & 1 \\
1 & 1 & 0 & 0 & 1 & 1 & 1 \\
1 & 1 & 0 & 0 & 1 & 1 & 1 \\
1 & 1 & 0 & 0 & 1 & 1 & 1 \\
1 & 1 & 0 & 0 & 1 & 1 & 1
\end{array}\right] \cdot\left[\begin{array}{l}
1 \\
1 \\
1 \\
1 \\
1 \\
1 \\
1
\end{array}\right]=[36]
$$

\section{Interpretation}

Each availability matrices $\left(S_{A}, S_{B}, S_{C}\right)$ display which individual system architecture elements have direct or indirect connection with each other. The availability grade (matrix' sum of all elements: $X_{A}, X_{B}, X_{C}$ ) indicates how many direct or indirect system connections are between the individual system architecture elements. According to the initial hypothesis: the more system connections exist, the better the system architectural layout is. The availability grade values $\left(X_{A}, X_{B}, X_{C}\right)$ answer the original question: which is the optimal system architecture layout? The higher availability grade signifies more available connections between the system architecture elements, and therefore the higher availability grade represents better system architecture layout. Because $X_{A}=4<X_{B}=17<X_{C}=36 \Rightarrow X_{C}$ identifies the best architectural layout, which is attributable to Case $\mathrm{C}$. This result justifies the initial hypothesis: the system architecture relying upon the centralized 
OTI-HS element is the optimal. This result means, that the centralized OTI-HSbased system is the optimal, and it is significantly better, than the legacy system architecture.

Adjacency matrix Availability matrix $\left(S_{X}\right) \quad$ Av. grade

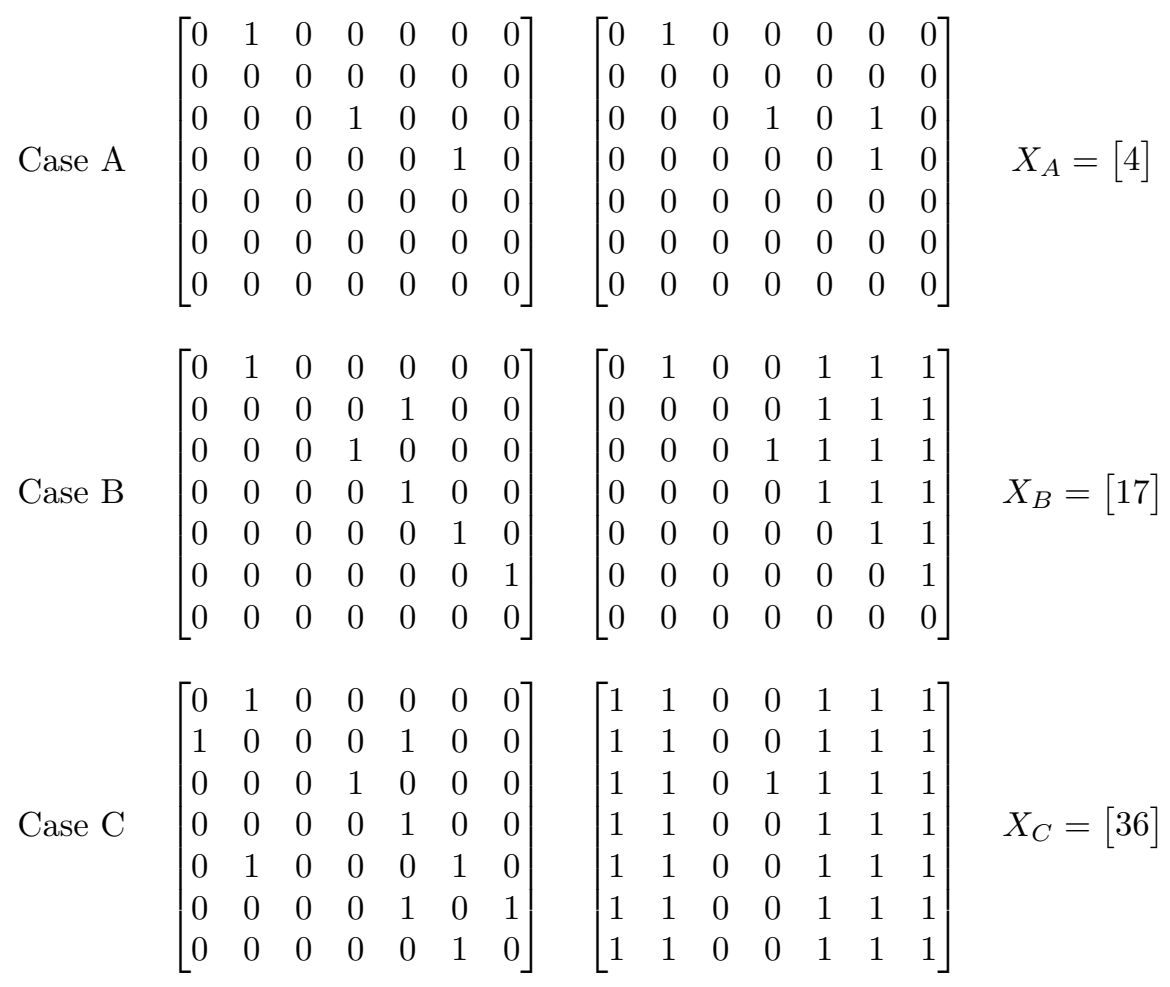

\section{Threats to validity}

The presented methodology applies mathematical calculations based on abstract service layout values. Exact, measurable performance indicators (response time, network speed) are not included in the method, which limits its universal applicability.

\section{Conclusion and Future Work}

This paper described a simplified demonstration of comparing three different cloudbased system architecture abstraction models. The initial hypothesis assumed that the architectural layout depending on a central element is more efficient than the one without it, because the central hub-component enables more connection among 
the system artifacts. The results satisfied the hypothesis. Therefore, it is also proved, that the central OTI-HS element enhances the overall system architecture performance. Consequently, the central OTI-HS component leads to optimized cloud-based e-Health and telemedicine system interoperability framework. So, we optimized the telemedicine and e-Health system architecture and interoperability framework with the OTI-HS. The calculations within this paper rely upon the given architecture abstraction-level. We will apply this demonstrated methodology for detailed-level architecture-design in the next research phase.

\section{References}

[1] The NIST Definition of Cloud Computing, Special Publication 800-145. National Institute of Standards and Technology, U.S. Department of Commerce, Gaithersburg (2011).

[2] VoAs, J., Network of 'Things', Special Publication 800-183. National Institue of Standards and Technology, U.S. Department of Commerce, Gaithersburg (2011).

[3] http://www. who.int/ehealth/en/ (last visited on 29.5.2017).

[4] Weiser, M., The Computer for the Twenty-First Century, Scientific American, Vol. 265/3 (1991), 94-104.

[5] NIST Big Data Interoperability Framework: Volume 1, Definitions, Special Publication 1500-1, National Institute of Standards and Technology, U.S. Department of Commerce, Gaithersburg (2015).

[6] Garai A., Empirical and Practical Implementation Methodology of E-Health IoT Technology, International Journal of Medical and Health Sciences Research, Vol. 3 Issue 12 (2016), 117-125.

[7] Garai A., Pentek I., Adamko A., Nemeth. A., Methodology for clinical integration of e-Health sensor-based smart device technology with cloud architecture, Pollack Periodica, Vol. 12, Issue 1 (2017), 69-80.

[8] Garai, A., Pentek, I., Adamko A., Nemeth A., Clinical System Integration Methodology for Bio-Sensory Technology with Cloud Architecture, Acta Cybernetica, accepted for publication in 2017.

[9] Gill, P. E., Murray, W., Wright, M. H., Practical Optimization, Emerald Group Publishing Limited (1982).

[10] Otto, C., Milenkonic, A., Sanders, C., Jovanov, E, System Architecture of a Wireless Body Area Sensor Network for Ubiquitous Health Monitoring, Journal of Mobile Multimedia, Vol. 1. No. 4. (2006), 307-326.

[11] Carson, R. S, Global System Architecture Optimization: Quantifying System Complexity, INCOSE International Symposium, Vol. 10 (2000), 416-421.

[12] Fischi, J., Nilchiani, R., Wade, J, Dynamic Complexity Measures for Use in Complexity-Based System Design, IEEE Systems Journal, Vol. PP, Issue 99 (2015).

[13] Pokoradi, L., Rendszerek es folyamatok modellezése, Campus kiadó, Debrecen (2008). 